\title{
A three year old girl was removed from her parents because of failure to recognize Mongolian spots
}

\author{
Søren Christoffersen
}

Institute of Forensic Medicine, Faculty of Health Sciences, University of Southern Denmark. J.B. Winslowsvej 17B, 5000 Odense C, Denmark

schristoffersen@health.sdu.dk

\begin{abstract}
:
A three year old girl was observed to have bluish marks on her back, arms and legs. The social services initiated an examination on the local hospital, but no conclusion regarding the marks was reached. The girl was institutionalized before a forensic examination after three days concluded that the marks were Mongolian spots. The case stresses the importance of being able to distinguish between Mongolian spots and skin hematomas and the need for a genuine clinical forensic examination in cases where child abuse is suspected. A guide for distinguishing between Mongolian spots and skin hematomas is presented.
\end{abstract}

Keywords:

Mongolian spots, skin hematomas, physical abuse, forensic examination

\section{INTRODUCTION}

The case presents the findings of discolored spots on a child who was suspected to have been the victim of physical abuse. Mongolian spots (MS) pose a well-known problem in the forensic field, or perhaps as the case shows, more a problem for doctors not used to dealing with clinical forensic medicine. In this case the inability to reach the right conclusion had severe consequences for the child to a degree luckily not seen very often.

\section{CASE REPORT}

A three and a half year old girl, born by Vietnamese parents, had started in her new kindergarten Monday morning. On Friday one of the employees, during a diaper change, noticed the girl had bluish marks on her buttocks, back and shoulders. Afraid that the marks were hematomas due to physical abuse the kindergarten contacted the social welfare service. Consequently the child was taken to the nearest larger hospital with a specialized children's ward for examination.

At the examination the staff found: blue spots in the buttocks area, on the back, shoulders, arms and legs, areas with light eczema on the arms and legs, generally dry skin and various scratch marks on the arms, legs and torso.

The diagnose MS was discussed but no decision was reached, the need for an examination by a forensic specialist was also discussed but not requested. The social services had had prior contact with the family where the mother had admitted to slapping the girl on the back of the head during eating when the girl was acting out, and the social services found that the connection between child and parents was off.

Due to the findings and prior history the social services wanted to remove the child from the home, the parents did however not want to give their consent. Presented with the ultimatum of a forceful removal of the girl the parents accepted and left the girl in the care of the social services. Later in the evening the parents returned to the hospital to see the girl only to find she had been moved to an institution, without anyone telling them.
Not until Monday morning when a police officer used to dealing with cases of child abuse came on duty, was a forensic examination requested. The examination yielded the same observations as those found at admittance to the hospital on Friday, (Figure 1), however there was no doubt the blue colored spots were Mongolian spots and not lesions caused by physical abuse. Still the girl was not returned to her parents until Tuesday.

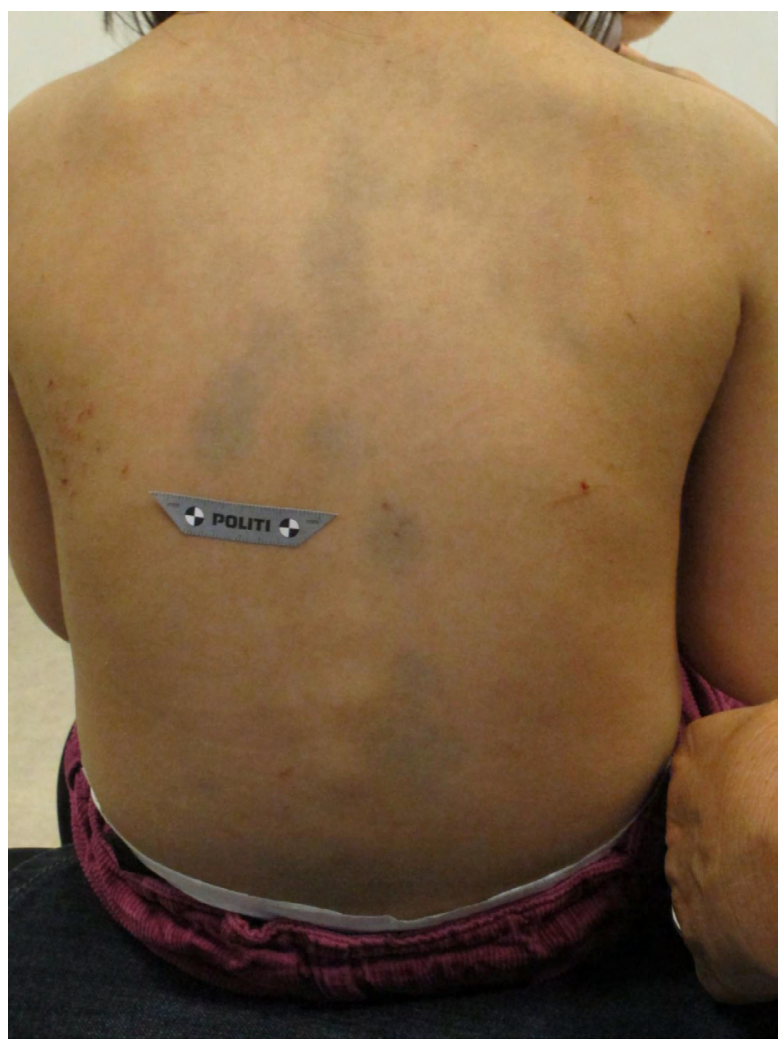

Figure 1. Blue-greyish spots in the sacral region and upper back, as presented at examination. 


\section{DISCUSSION}

Histologically Mongolian spots are characterized by spindle-shaped melanocytes in the dermis, believed to derive from failed migration of melanocytes from the neural crest to the epidermis in fetal life [1]. The spots are typically present at birth or appear shortly hereafter, though late onset has been observed as late as the age of 262

Mongolian spots are equally common in both sexes and can be observed in all races. They are most commonly seen in Asians and Africans, present in up to $100 \%$ of newborns in some studies. Also other ethnic groups with dark skin such as Eskimoes, North American Indians, South Americans and Middle Easterners have a high frequency. The spots are less commonly observed in Caucasians [3-5].

The color varies from light grey over blue-grey and greenish to almost completely black. MS may be found all over the body. Most commonly affected area is the sacral region, different studies report slightly varying most common extra-sacral location, but back and extremities are second to the sacral region, least common is facial location [5-8].

Shape and size vary, but typically the elements are irregular or oval and not larger than $20 \mathrm{~cm} \emptyset$. The spots usually disappear within the first year of life and are rarely found after the age of six, though they may persist through adult life [5]. MS can be superimposed which might imitate a bruise in a MS affected area [9].

Besides various other hyperpigmentation skin conditions, an important and well known differential diagnosis of MS is physical abuse [10]. MS do however differentiate from skin hematomas with regard to color, in two important aspects. MS are typically quite uniform both inter and intra elemental whereas skin hematomas will present color variation. The color variation is most often quite noticeable between the various lesions and even more so within the single elements. The color of the center of a hematoma will typically differentiate from the edge, and depending on the age of a skin hematoma, you would expect to see a mix of colors, from red, blue, green, brown and yellow, (Figure 2).

In the case presented, a three year old girl was institutionalized for more than three days because MS were not recognized. Possibly because the doctor was not able to diagnose MS compared to skin hematomas. Secondly, the examiner might have been influenced by the social services who already knew the family and had an agenda of their own. If the doctor acted due to a wish from the social services, it might have been with good intensions. However such a practice would only serve to undermine the value of medical examinations in cases of child abuse and the impartialness of medical doctors. In my opinion, it is safe to say that even if this little girl was indeed exposed to physical abuse in her home, the degree of violence was not in any way life-threatening, and she would have been better off staying at home during the weekend than being removed from home and placed in an institution.

Whichever of the possibilities is the correct, a medical doctor should, in our opinion, be able to distinguish MS from skin hematomas, even more so as staff on a specialized children's ward where MS would be a common occurrence.

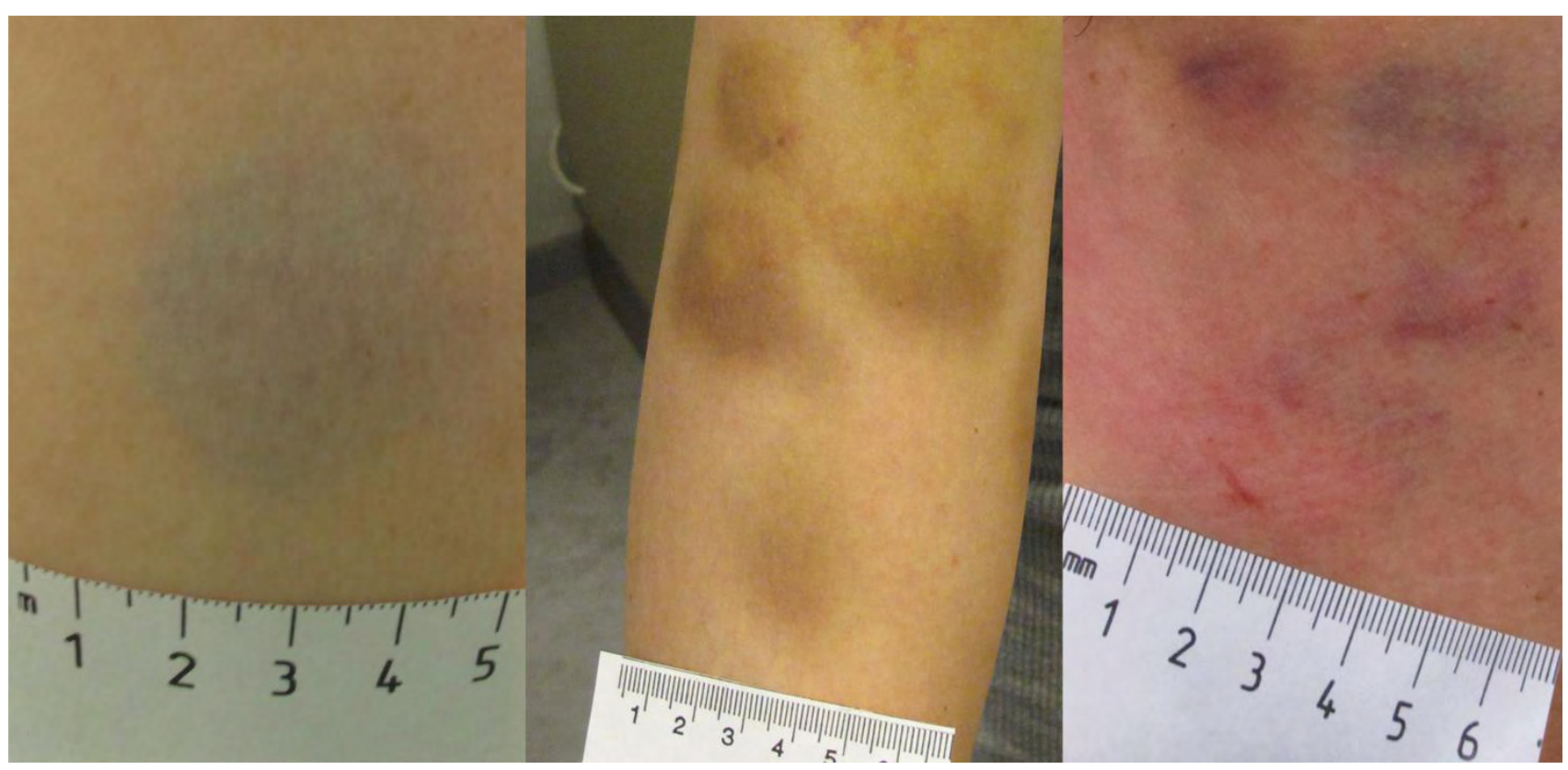

Figure 2. Color variation in skin hematomas. 


\section{REFERENCES}

[1] Musumeci ML, Lacarrubba F, Santagati C, Micali G. Multiple and superimposed Mongolian spots. BMJ case reports. 2013;2013.

[2] Carmichael AJ, Tan CY, Abraham SM. Adult onset Mongolian spot. Clinical and experimental dermatology. 1993;18(1):72-74.

[3] Cordova A. The Mongolian spot: a study of ethnic differences and a literature review. [Clinical pediatrics. 1981;20(11):714-719.

[4] Gupta D, Thappa DM. Mongolian spots: How important are they? World journal of clinical cases. 2013;1(8):230-232.

[5] Gupta D, Thappa DM. Mongolian spots. Indian journal of dermatology, venereology and leprology. 2013;79(4):469-478.

[6] Gupta D, Thappa DM. Mongolian Spots-A Prospective Study. Pediatric dermatology. 2013.
[7] Egemen A, Ikizoglu T, Ergor S, Mete Asar G, Yilmaz O. Frequency and characteristics of mongolian spots among Turkish children in Aegean region. The Turkish journal of pediatrics. 2006;48(3):232-236.

[8] Reza AM, Farahnaz GZ, Hamideh S, Alinaghi SA, Saeed Z, Mostafa H. Incidence of Mongolian spots and its common sites at two university hospitals in Tehran, Iran. Pediatric dermatology. 2010;27(4):397-398.

[9] Leung AK, Robson WL. Superimposed Mongolian spots. Pediatric dermatology. 2008;25(2):233-235.

[10] AlJasser M, Al-Khenaizan S. Cutaneous mimickers of child abuse: a primer for pediatricians. European journal of pediatrics. 2008;167(11):1221-1230. 